\title{
Construction of a Frailty Index as a Novel Health Measure in Systemic Lupus Erythematosus
}

\author{
Alexandra Legge, Susan Kirkland, Kenneth Rockwood, Pantelis Andreou, Sang-Cheol Bae, \\ Caroline Gordon, Juanita Romero-Diaz, Jorge Sanchez-Guerrero, Daniel J. Wallace, \\ Sasha Bernatsky, Ann E. Clarke, Joan T. Merrill, Ellen M. Ginzler, Paul Fortin, \\ Dafna D. Gladman, Murray B. Urowitz, Ian N. Bruce, David A. Isenberg, Anisur Rahman, \\ Graciela S. Alarcón, Michelle Petri, Munther A. Khamashta, M.A. Dooley, \\ Rosalind Ramsey-Goldman, Susan Manzi, Asad A. Zoma, Cynthia Aranow, Meggan Mackay, \\ Guillermo Ruiz-Irastorza, S. Sam Lim, Murat Inanc, Ronald F. van Vollenhoven, \\ Andreas Jonsen, Ola Nived, Manuel Ramos-Casals, Diane L. Kamen, Kenneth C. Kalunian, \\ Soren Jacobsen, Christine A. Peschken, Anca Askanase, and John G. Hanly
}

\begin{abstract}
Objective. To construct a Frailty Index (FI) as a measure of vulnerability to adverse outcomes among patients with systemic lupus erythematosus (SLE), using data from the Systemic Lupus International Collaborating Clinics (SLICC) inception cohort.

Methods. The SLICC inception cohort consists of recently diagnosed patients with SLE followed annually with clinical and laboratory assessments. For this analysis, the baseline visit was defined as the first study visit at which sufficient information was available for construction of an FI. Following a standard procedure, variables from the SLICC database were evaluated as potential health deficits. Selected health deficits were then used to generate a SLICC-FI. The prevalence of frailty in the baseline dataset was evaluated using established cutpoints for FI values.

Results. The 1683 patients with SLE (92.1\% of the overall cohort) eligible for inclusion in the baseline dataset were mostly female (89\%) with mean (SD) age 35.7 (13.4) years and mean (SD) disease duration 18.8 (15.7) months at baseline. Of 222 variables, 48 met criteria for inclusion in the SLICC-FI. Mean (SD) SLICC-FI was $0.17(0.08)$ with a range from 0 to 0.51 . At baseline, $27.1 \%$ (95\% CI 25.0-29.2) of patients were classified as frail, based on SLICC-FI values $>0.21$.

Conclusion. The SLICC inception cohort permits feasible construction of an FI for use in patients with SLE. Even in a relatively young cohort of patients with SLE, frailty was common. The SLICC-FI may be a useful tool for identifying patients with SLE who are most vulnerable to adverse outcomes, but validation of this index is required prior to its use. (First Release August 15 2019; J Rheumatol 2020;47:72-81; doi:10.3899/jrheum.181338)
\end{abstract}

Key Indexing Terms: SYSTEMIC LUPUS ERYTHEMATOSUS OUTCOME ASSESSMENT COHORT STUDIES

\begin{abstract}
From the Divisions of Rheumatology and Geriatric Medicine, Department of Medicine, Dalhousie University; Department of Community Health and Epidemiology, Dalhousie University, Halifax, Nova Scotia; Divisions of Rheumatology and Clinical Epidemiology, Department of Medicine, McGill University, Montreal; Division of Rheumatology, Centre Hospitalier Universitaire (CHU) de Québec et Université Laval, Quebec City, Quebec; Division of Rheumatology, Cumming School of Medicine, University of Calgary, Calgary, Alberta; Center for Prognosis Studies in the Rheumatic Diseases, Toronto Western Hospital and University of Toronto, Toronto, Ontario; University of Manitoba, Winnipeg, Manitoba; Division of Rheumatology, Department of Medicine and Department of Pathology, Queen Elizabeth II Health Sciences Center and Dalhousie University, Halifax, Nova Scotia, Canada; Department of Rheumatology, Hanyang University Hospital for Rheumatic Diseases, Seoul, Korea; Rheumatology Research Group, School of Immunity and Infection, College of Medical and Dental Sciences, University of Birmingham, Birmingham; Arthritis Research UK Epidemiology Unit, Faculty of Biology Medicine and Health, Manchester Academic Health Sciences Center, The University of Manchester, and National Institute for Health Research (NIHR) Manchester Musculoskeletal Biomedical Research Centre, Manchester University National Health Service (NHS) Foundation Trust, Manchester
\end{abstract}

\begin{abstract}
Academic Health Science Center, Manchester; Center for Rheumatology, Department of Medicine, University College London, London; Lanarkshire Center for Rheumatology, Hairmyres Hospital, East Kilbride, Scotland; Lupus Research Unit, The Rayne Institute, St Thomas' Hospital, King's College London School of Medicine, London, UK; Instituto Nacional de Ciencias Medicas y Nutrición, Mexico City, Mexico; Cedars-Sinai/David Geffen School of Medicine at University of California Los Angeles (UCLA), Los Angeles, California; Department of Clinical Pharmacology, Oklahoma Medical Research Foundation, Oklahoma City, Oklahoma; Department of Medicine, State University of New York (SUNY) Downstate Medical Center, Brooklyn, New York; Department of Medicine, University of Alabama at Birmingham, Birmingham, Alabama; Division of Rheumatology, Johns Hopkins University School of Medicine, Baltimore, Maryland; Thurston Arthritis Research Center, University of North Carolina, Chapel Hill, North Carolina; Northwestern University and Feinberg School of Medicine, Chicago, Illinois; Lupus Center of Excellence, Allegheny Health Network, Pittsburgh, Pennsylvania; Feinstein Institute for Medical Research, Manhasset, New York; Emory University School of Medicine, Division of Rheumatology, Atlanta, Georgia; Medical University of South Carolina, Charleston, South Carolina; University of California San Diego School of Medicine (UCSD),
\end{abstract}


La Jolla, California; Hospital for Joint Diseases, New York University (NYU), Seligman Center for Advanced Therapeutics, New York, New York, USA; Autoimmune Diseases Research Unit, Department of Internal Medicine, BioCruces Health Research Institute, Hospital Universitario Cruces, University of the Basque Country, Barakaldo; Josep Font Autoimmune Diseases Laboratory, IDIBAPS, Department of Autoimmune Diseases, Hospital Clínic, Barcelona, Spain; Division of Rheumatology, Department of Internal Medicine, Istanbul Medical Faculty, Istanbul University, Istanbul, Turkey; Unit for Clinical Therapy Research (ClinTRID), Karolinska Institute, Stockholm; Department of Clinical Sciences Lund, Rheumatology, Lund University, Lund, Sweden; Copenhagen Lupus and Vasculitis Clinic, Section 4242, Rigshospitalet, Copenhagen University Hospital, Copenhagen, Denmark.

Funding for this work was provided by the Nova Scotia Health Authority Research Fund. Dr. Bae's work was supported in part by NRF-2017M3A9B4050335, Republic of Korea. Dr. Gordon's work was supported by Lupus UK and the NIHR/Wellcome Trust Clinical Research Facility. The Montreal General Hospital Lupus Clinic is partially supported by the Singer Family Fund for Lupus Research. Dr. Clarke holds The Arthritis Society Chair in Rheumatic Diseases at the University of Calgary. Dr. Fortin presently holds a tier 1 Canada Research Chair on Systemic Autoimmune Rheumatic Diseases at Université Laval, and part of this work was done while he was still a Distinguished Senior Investigator of The Arthritis Society. Dr. Bruce is an NIHR Senior Investigator and is supported by Arthritis Research UK, the NIHR Manchester Biomedical Centre, and the NIHR/Wellcome Trust Manchester Clinical Research Facility. The views expressed in this publication are those of the author(s) and not necessarily those of the NHS, the NIHR, or the Department of Health. Dr. Isenberg and Dr. Rahman are supported by the NIHR University College London Hospitals Biomedical Research Center. The Hopkins Lupus Cohort is supported by the US National Institutes of Health (NIH; grant AR43727 and 69572). Dr. Dooley's work was supported by the NIH (grant RR00046). Dr. Ramsey-Goldman's work was supported by the NIH (grants 5UL1TR001422-02, formerly 8UL1TR000150 and UL-1RR-025741, K24-AR-02318, and P60AR064464 formerly P60-AR-48098). Dr. Ruiz-Irastorza is supported by the Department of Education, Universities and Research of the Basque Government. Dr. Jacobsen is supported by the Danish Rheumatism Association (A3865) and the Novo Nordisk Foundation (A05990). Dr. Hanly is supported by the Canadian Institutes of Health Research (grant MOP-88526).

A. Legge, MD, Department of Medicine, Division of Rheumatology, Dalhousie University; S. Kirkland, PhD, Department of Community Health and Epidemiology, Dalhousie University; K. Rockwood, MD Division of Geriatric Medicine, Department of Medicine, Dalhousie University; P. Andreou, PhD, Department of Community Health and Epidemiology, Dalhousie University; S.C. Bae, MD, Department of Rheumatology, Hanyang University Hospital for Rheumatic Diseases; C. Gordon, MD, Rheumatology Research Group, School of Immunity and Infection, College of Medical and Dental Sciences, University of Birmingham; J. Romero-Diaz, MD, Instituto Nacional de Ciencias Medicas y Nutrición; J. Sanchez-Guerrero, MD, Instituto Nacional de Ciencias Medicas y Nutrición; D.J. Wallace, MD, Cedars-Sinai/David Geffen School of Medicine at UCLA; S. Bernatsky, MD, Divisions of Rheumatology and Clinical Epidemiology, Department of Medicine, McGill University; A.E. Clarke, MD, Division of Rheumatology, Cumming School of Medicine, University of Calgary; J.T. Merrill, MD, Department of Clinical Pharmacology, Oklahoma Medical Research Foundation; E.M. Ginzler, MD, Department of Medicine, SUNY Downstate Medical Center; P. Fortin, MD, Division of Rheumatology, CHU de Québec et Université Laval, D.D. Gladman, MD, Center for Prognosis Studies in the Rheumatic Diseases, Toronto Western Hospital and University of Toronto, M.B. Urowitz, MD, Center for Prognosis Studies in the Rheumatic

Diseases, Toronto Western Hospital and University of Toronto; I.N. Bruce, MD, Arthritis Research UK Epidemiology Unit, Faculty of Biology

Medicine and Health, Manchester Academic Health Sciences Center, The University of Manchester, and NIHR Manchester Musculoskeletal Biomedical Research Centre, Manchester University NHS Foundation Trust, Manchester Academic Health Science Center; D.A. Isenberg, MD, Center for Rheumatology, Department of Medicine, University College London; A. Rahman, MD, Center for Rheumatology, Department of Medicine, University College London; G.S. Alarcón, MD, Department of
Medicine, University of Alabama at Birmingham; M. Petri, MD, Division of Rheumatology, Johns Hopkins University School of Medicine; M.A. Khamashta, MD, Lupus Research Unit, The Rayne Institute, St Thomas' Hospital, King's College London School of Medicine; M.A. Dooley, MD, Thurston Arthritis Research Center, University of North Carolina; R. Ramsey-Goldman, MD, Northwestern University and Feinberg School of Medicine; S. Manzi, MD, Lupus Center of Excellence, Allegheny Health Network; A.A. Zoma, MD, Lanarkshire Center for Rheumatology, Hairmyres Hospital; C. Aranow, MD, Feinstein Institute for Medical Research; M. Mackay, MD, Feinstein Institute for Medical Research; G. Ruiz-Irastorza, MD, Autoimmune Diseases Research Unit, Department of Internal Medicine, BioCruces Health Research Institute, Hospital Universitario Cruces, University of the Basque Country; S.S. Lim, MD, Emory University School of Medicine, Division of Rheumatology; M. Inanc, MD, Division of Rheumatology, Department of Internal Medicine, Istanbul Medical Faculty, Istanbul University; R.F. van Vollenhoven, MD, ClinTRID, Karolinska Institute; A. Jonsen, $M D$, Department of Clinical Sciences Lund, Rheumatology, Lund University; O. Nived, MD, Department of Clinical Sciences Lund, Rheumatology, Lund University; M. Ramos-Casals, MD, Josep Font Autoimmune Diseases Laboratory, IDIBAPS, Department of Autoimmune Diseases, Hospital Clinic; D.L. Kamen, MD, Medical University of South Carolina; K.C. Kalunian, MD, UCSD School of Medicine; S. Jacobsen, MD, Copenhagen Lupus and Vasculitis Clinic, Section 4242,

Rigshospitalet, Copenhagen University Hospital; C.A. Peschken, MD, University of Maniptoba; A. Askanase, MD, Hospital for Joint Diseases, NYU, Seligman Center for Advanced Therapeutics; J.G. Hanly, MD, Division of Rheumatology, Department of Medicine and Department of Pathology, Queen Elizabeth II Health Sciences Center and Dalhousie University.

Address correspondence to Dr. J.G. Hanly, Division of Rheumatology, Nova Scotia Rehabilitation Centre (2nd Floor), 1341 Summer St., Halifax, Nova Scotia B3H 4K4, Canada.E-mail: john.hanly@nshealth.ca Accepted for publication March 27, 2019.

Systemic lupus erythematosus (SLE) is an autoimmune disease with diverse manifestations and an unpredictable clinical course ${ }^{1}$. Despite advances in diagnosis and treatment ${ }^{2}$, many patients with SLE accumulate organ damage $^{3}$, and the mortality risk remains high ${ }^{4,5}$. Given this variability in health trajectories, it would be advantageous to identify those patients with SLE at increased risk for adverse outcomes. However, instruments that accurately predict longterm outcomes in SLE are limited ${ }^{6}$.

In geriatric medicine ${ }^{7}$, and increasingly in other disciplines $8,9,10,11,12,13$, differences in susceptibility to adverse outcomes are quantified using the construct of frailty, defined as a state of increased vulnerability due to degradation of homeostatic mechanisms, resulting in diminished ability to respond to physiologic stressors ${ }^{14}$. Although often linked to advanced age, frailty can be observed across the life course ${ }^{15}$, including among individuals with acquired vulnerability states $^{16,17}$

Two different conceptual approaches inform the measurement of frailty ${ }^{18}$. One approach uses rules-based tools, where specific criteria must be met to classify an individual as frail ${ }^{18}$. The most common example of this approach is the Fried frailty phenotype, which defines frailty as a clinical syndrome with at least 3 of 5 specific health deficits: weight loss, exhaustion, physical inactivity, slow walking speed, and reduced grip strength ${ }^{19}$.

The second approach to measuring frailty is the Frailty 
Index $(\mathrm{FI})^{20}$, which conceptualizes frailty as a loss of physiologic reserve arising from the accumulation of health deficits across multiple systems ${ }^{21}$. Individuals who possess few deficits are considered relatively fit, while those with an increasing number of health problems are considered increasingly frail ${ }^{18}$. Prior studies have consistently shown an association between higher FI values and increased risk of negative health outcomes, including hospitalizations, morbidity, and mortality $15,22,23,24$. Although used in many different clinical contexts $^{22,23,25}$, the deficit accumulation approach has yet to be applied in SLE.

Health deficits in SLE may occur because of the disease, its treatment, other comorbidities, or aging. Evaluating frailty through deficit accumulation could improve our understanding of the heterogeneous health outcomes in SLE. The aim of the present study was to use the deficit accumulation approach to construct an FI as a novel health measure in SLE, using data from an international inception cohort. Future studies are required to validate the Systemic Lupus International Collaborating Clinics (SLICC)-FI, including its predictive validity for adverse health outcomes.

\section{MATERIALS AND METHODS}

Data source. This was a secondary analysis of longitudinal data from the SLICC inception cohort. SLICC comprises 52 investigators at 43 academic centers in 16 countries. From 1999 to 2011, a cohort of 1826 recently diagnosed patients with SLE was recruited from 31 SLICC sites in Europe, Asia, and North America. Patients were enrolled within 15 months of SLE diagnosis, based on $\geq 4$ revised American College of Rheumatology (ACR) classification criteria for $\mathrm{SLE}^{26}$. At enrollment and annually thereafter, data were collected per a standardized protocol, submitted to the coordinating centers at the University of Toronto (Toronto, Ontario, Canada) and Dalhousie University (Halifax, Nova Scotia, Canada), and entered into centralized databases. The study was approved by the institutional research ethics boards of the Nova Scotia Health Authority central zone (\#1020396) and of participating centers in accordance with the Declaration of Helsinki's guidelines for research in humans. All participants provided written informed consent.

Clinical assessments. Demographic features included age, sex, race/ethnicity, geographic location, and years of post-secondary education. Corticosteroid, antimalarial, and immunosuppressive use was documented. Medical comorbidities prior to SLE diagnosis and between followup visits were recorded. The revised ACR classification criteria for $\mathrm{SLE}^{26}$ and neuropsychiatric events ${ }^{27}$ were documented at enrollment and between followup visits ${ }^{28}$. SLE disease activity was measured using the SLE Disease Activity Index $2000^{29}$, cumulative organ damage using the SLICC/ACR Damage Index (SDI) ${ }^{30}$, and health-related quality of life using the Medical Outcomes Survey Short Form-36 (SF-36) ${ }^{31}$. Blood pressure (in mmHg), height (m), and weight $(\mathrm{kg})$ were also recorded.

Laboratory data. Investigations for the assessment of SLE disease activity and organ damage were performed at each visit: anti-dsDNA, C3 and C4, serum creatinine, urinalysis, fasting glucose, lipid profile, and inflammatory markers (erythrocyte sedimentation rate and C-reactive protein).

Standard procedure for FI construction. An FI can be constructed from any existing health dataset using a standard procedure described by Searle, et al (Table 1$)^{20}$. These methods have been shown to be valid and reliable ${ }^{15,22,23,32,33,34}$. Briefly, potential health deficits are first identified. A health deficit is any symptom, physical sign, disease process, functional impairment, or laboratory abnormality that is acquired, associated with adverse health outcomes, and associated with chronological age ${ }^{20,35}$. If
Table 1. Standard criteria for the identification of health deficits for inclusion in a frailty index.

Standard Criteria

Health deficit definition

Any symptom, physical sign, disease process, functional impairment, or laboratory/radiographic abnormality

Criteria to be met by each individual health deficit

1. Must be acquired, as opposed to innate

2. Must be associated with an adverse health outcome

3. Prevalence should generally increase with increasing chronological age

4. Must be present in at least $1 \%$, but not more than $80 \%$ of the sample

5. Must have non-missing values for at least $95 \%$ of the sample

Criteria to be met by the overall set of health deficits

1. Must cover a range of physiologic organ systems

2. Must include integrated variables indicative of repair potential, including measures of function and mobility

3. Must include at least $30-40$ deficits in total

deficits are either too infrequent or too common, they are unlikely to provide meaningful information in an FI, and are respectively combined or excluded $^{20,35}$. Finally, if a single item is missing values for $>5 \%$ of individuals, it is excluded ${ }^{20,35}$.

The totality of health deficits in an FI must represent several organ systems. Of note, frailty not only measures irreversible damage but also measures an individual's potential for recovery. Therefore, an FI also includes measures of function and mobility 20,35 . Finally, an FI requires a minimum of $30-40$ health deficits to produce stable and precise estimates of frailty $22,33,35,36$.

Each health deficit is assigned a score from 0 to 1 , with 0 representing no deficit and 1 representing the deficit fully expressed ${ }^{20}$. Health deficit scores are combined to produce an FI score between 0 and 1, calculated as the sum of deficit scores for an individual divided by the total number of deficits considered ${ }^{20,35}$

Establishing a baseline dataset for SLICC-FI construction. Given the importance of the SDI and the SF-36 for the construction of the SLICC-FI, each patient's baseline visit was defined as the first at which both an SDI and an SF-36 were completed. Patients were excluded if they had never had an SDI recorded, never had an SF-36 recorded, or never had both instruments recorded at the same visit.

Selecting health deficits for the SLICC-FI. Potential health deficits were evaluated using the criteria in Table 1. Variables judged to be innate, as opposed to acquired, were excluded. Age-relatedness was assessed by reviewing the literature to determine whether each variable is observed more frequently with increasing age in SLE populations. While a health deficit should generally increase in prevalence with increasing age, this relationship may not exist for all deficits, in part because of survivor effects ${ }^{20}$. Variables were retained in the SLICC-FI even if there was attenuation of this relationship at advanced ages.

The association of each health deficit with increased risk of adverse health outcomes in SLE was also determined through literature review. Variables not clearly associated with adverse outcomes were excluded. If literature specific to SLE was not available, evidence from the general population was sought and extrapolated to SLE populations.

Next, variables were evaluated for duplications. Items were excluded from the SLICC-FI if they represented constructs that were already better accounted for by another variable in the database. Where appropriate, multiple related variables were combined to produce single health deficits. Variables whose prevalence in the dataset was $<1 \%$ were excluded if there were no similar deficits with which they could be reasonably combined. Finally, variables were excluded if their prevalence in the dataset was $>80 \%$, or if there were missing values for $>5 \%$ of observations. 
Coding of individual health deficits for the SLICC-FI. Binary variables were assigned a score of 0 (absence of the deficit) or 1 (presence of the deficit). Ordinal variables were coded by converting the number of possible ranks into equally spaced scores ranging from 0 to 1 . Continuous variables were coded using established cutpoints from the SLE literature.

SLICC-FI calculation. Individual health deficit scores were combined to produce a SLICC-FI score for each patient. For example, with 48 health deficits in the SLICC-FI, an individual in whom 24 of these deficits were fully present would have a SLICC-FI score of 24/48 $=0.50$. SLICC-FI scores were not calculated for individuals with missing values for $>20 \%$ of health deficits $^{36}$.

Statistical analysis. Descriptive statistics were calculated for demographic and clinical characteristics. For quantitative variables, measures of central tendency (means and medians) and dispersion (SD and interquartile ranges) were reported, as appropriate. For categorical variables, absolute and relative frequencies were reported. Descriptive statistics were calculated for SLICC-FI values and the distribution of SLICC-FI scores was visualized. Using cutpoints derived in the general population ${ }^{15,37,38}$, we classified patients as robust $($ SLICC-FI $\leq 0.03)$, relatively less fit $(0.03<$ SLICC-FI $\leq 0.10)$, least fit $(0.10<$ SLICC-FI $\leq 0.21)$, or frail $($ SLICC-FI $>0.21)$, and reported the prevalence of frailty with $95 \% \mathrm{CI}$.

To evaluate for bias due to varying SLE disease durations, analyses were repeated in patients with baseline assessments within 2 years of SLE diagnosis. Finally, to evaluate the effect of a given variable on the SLICC-FI, an iterative, resampling procedure was used ${ }^{20,39}$. One hundred iterations were performed in which each iteration calculated SLICC-FI values using $80 \%$ of health deficits and then reevaluated the descriptive statistics of the SLICC-FI. Data analysis was conducted using STATA-IC Version 14 (StataCorp).

\section{RESULTS}

Patient characteristics. There were 1683 patients (92.2\% of cohort) with study visits at which both the SDI and SF-36 were recorded. The first such visit was included in our baseline dataset, and for most patients this occurred early in their disease course [1390/1683 patients (82.6\%) within $2 \mathrm{yrs}$ of SLE diagnosis]. Demographic and clinical characteristics are shown in Table 2.

Excluded patients. There were 143 patients (7.8\% of cohort) excluded, most $(\mathrm{n}=90)$ of whom had a single visit within 6 months of diagnosis, which precluded scoring the SDI. Other reasons for exclusion were no SF-36 recorded $(n=32)$, no SDI recorded $(n=6)$, and no visit with both SF-36 and SDI recorded $(n=15)$. At enrollment, excluded patients were similar to non-excluded patients in age, sex, education, SLE disease activity, and SLE manifestations (data not shown). Hispanic patients were more likely to be excluded compared to patients of other races/ethnicities, largely owing to higher rates of missing SF-36 data and early loss to followup (data not shown).

SLICC-FI construction: selection of health deficits. Of the 222 candidate variables identified as potential health deficits (Figure 1), 18 were excluded for failing to meet the first 3 health deficit criteria (Table 1) and 46 were excluded as duplicates. The remaining 158 SLICC variables were used to construct health deficits. There were 36 variables that were directly converted into 36 health deficits. In other cases, several variables representing varying aspects of the same
Table 2. Baseline characteristics of SLICC inception cohort patients included in the dataset for SLICC-FI construction $(\mathrm{n}=1683)$.

\begin{tabular}{lc}
\hline Variables & Values \\
\hline Patient age at baseline, yrs, mean (SD) & $35.7(13.4)$ \\
Sex & \\
Female & $1493(88.7)$ \\
Male & $190(11.3)$ \\
Race/ethnicity & \\
White & $834(49.6)$ \\
African ancestry & $280(16.6)$ \\
Asian & $260(15.5)$ \\
Hispanic & $248(14.7)$ \\
Other & $61(3.6)$ \\
Geographic location & \\
USA & $467(27.7)$ \\
Canada & $395(23.5)$ \\
Mexico & $197(11.7)$ \\
Europe & $461(27.4)$ \\
Asia & $163(9.7)$ \\
Education & \\
Post-secondary education & $847(50.3)$ \\
Missing & $22(1.3)$ \\
Cigarette smoking, current & $242(14.4)$ \\
SLE disease duration at baseline, mos, median (IQR) & $14.0(10.7-18.4)$ \\
SLEDAI-2K at baseline, median (IQR) & $2(0-6)$ \\
Baseline SDI = 0 & $1270(75.5)$ \\
Medication use & \\
Corticosteroids & $1179(70.1)$ \\
Antimalarials & $1149(68.3)$ \\
Immunosuppressives & $681(40.5)$ \\
\hline
\end{tabular}

Values are $\mathrm{n}(\%)$ unless otherwise specified. IQR: interquartile range; SLICC: Systemic Lupus International Collaborating Clinics; FI: Frailty Index; SLE: systemic lupus erythematosus; SLEDAI-2K: SLE Disease Activity Index 2000; SDI: SLICC/American College of Rheumatology Damage Index.

condition were combined to create a single health deficit. For example, the health deficit "coronary artery disease," defined as "any history of angina, myocardial infarction, or coronary revascularization ever," used information from 12 different variables. Thus, information from the remaining 122 variables was combined to form 32 health deficits. In total, 68 distinct health deficits were generated for further evaluation. Of these, 9 were excluded owing to low baseline prevalence $(<1 \%)$, one owing to high baseline prevalence $(>80 \%)$, and 10 because of missing data in $>5 \%$ of observations. Forty-eight health deficits met all required criteria for inclusion in the SLICC-FI.

SLICC-FI construction: health deficit coding. The majority of SLICC-FI health deficits were binary, with values of either 0 or 1. Examples included "diabetes" and "active nephritis." Ordinal health deficits included those derived from the SF-36. For example, for "self-rated health," the self-reported SF-36 responses were coded as "excellent $=0$," "very good $=0.25$," "good $=0.5$," "fair $=0.75$," and "poor $=1$." For continuous variables, existing literature was used to define clinically significant cutpoints. For example, the "body mass index" (BMI) cutpoints were derived from published data

Personal non-commercial use only. The Journal of Rheumatology Copyright (C) 2019. All rights reserved. 


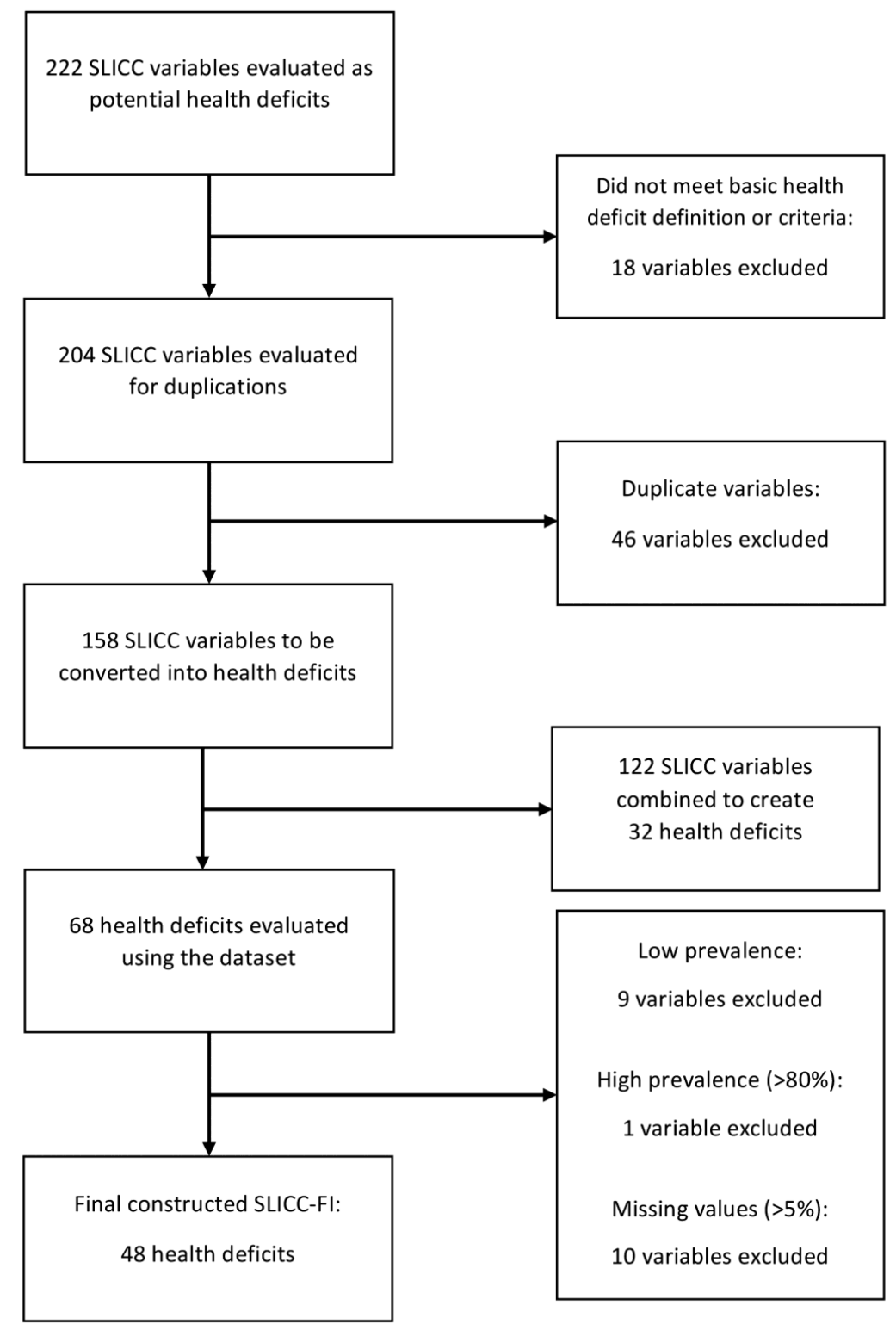

Figure 1. Flow diagram of the evaluation of SLICC variables for inclusion as health deficits in the SLICC-FI. SLICC: Systemic Lupus International Collaborating Clinics; FI: Frailty Index.

regarding the association between BMI and mortality in the general population (BMI $18.5-24.9 \mathrm{~kg} / \mathrm{m}^{2}=0$; BMI $25-29.9$ $\mathrm{kg} / \mathrm{m}^{2}=0.5$; BMI $<18.5$ or $\left.\geq 30 \mathrm{~kg} / \mathrm{m}^{2}=1\right)^{40}$.

The SLICC-FI. Of the 48 health deficits in the SLICC-FI (Table 3, and Supplementary Table 1, available with the online version of this article), 14 were related to organ damage, before or after the diagnosis of SLE (e.g., congestive heart failure and chronic kidney disease). Another 14 deficits reflected active inflammation (e.g., serositis and inflammatory arthritis), while 6 items reflected comorbid conditions (e.g., hypertension and obesity). Finally, there were 14 variables related to function, mobility, health attitude, and mental health.

SLICC-FI values. SLICC-FI scores were calculated for 1682 patients in the baseline dataset. In 1 patient, a SLICC-FI score could not be calculated because of missing data for $12(25 \%)$ health deficits. The distribution of baseline SLICC-FI scores (Figure 2) ranged from 0 to 0.51 , with a median (IQR) of 0.16 (0.11-0.22) and a mean (SD) of $0.17(0.08)$.

Based on SLICC-FI values $>0.21,27.1 \%(95 \% \mathrm{CI}$ 25.0-29.2) of patients with SLE were classified as frail at baseline (Table 4). The prevalence of frailty increased with 
Table 3. Systemic Lupus International Collaborating Clinics-Frailty Index health deficits.

\begin{tabular}{|c|c|}
\hline Health Deficits & Scoring System \\
\hline Diabetes & $\mathrm{No}=0 ; \mathrm{Yes}=1$ \\
\hline Malignancy & No $=0 ;$ Yes $=1$ \\
\hline Coronary artery disease & No $=0 ;$ Yes $=1$ \\
\hline Congestive heart failure & No $=0 ;$ Yes $=1$ \\
\hline Peripheral vascular disease & $\mathrm{No}=0 ; \mathrm{Yes}=1$ \\
\hline Cerebrovascular disease & No $=0 ;$ Yes $=1$ \\
\hline Chronic kidney disease & $\begin{array}{l}\text { None }=0 ; \text { Stage } 1=0.2 ; \text { Stage } 2=0.4 ; \text { Stage } 3=0.6 ; \text { Stage } 4=0.8 \\
\text { Stage } 5=1\end{array}$ \\
\hline Deforming or erosive arthritis & $\mathrm{No}=0 ;$ Yes $=1$ \\
\hline Venous thromboembolism & No $=0 ;$ Yes $=1$ \\
\hline Pulmonary disease & No $=0 ;$ Yes $=1$ \\
\hline Gastrointestinal disease & $\mathrm{No}=0 ; \mathrm{Yes}=1$ \\
\hline Osteoporosis/avascular necrosis & $\mathrm{No}=0 ; \mathrm{Yes}=1$ \\
\hline Ocular manifestations related to SLE & No $=0 ;$ Yes $=1$ \\
\hline SLE myocarditis/endocarditis & $\mathrm{No}=0 ; \mathrm{Yes}=1$ \\
\hline Cognitive impairment & No $=0 ;$ Yes $=1$ \\
\hline Seizures and seizure disorders & No $=0 ;$ Yes $=1$ \\
\hline Altered mental status & $\mathrm{No}=0 ; \mathrm{Yes}=1$ \\
\hline Neuropathy & No $=0 ;$ Yes $=1$ \\
\hline Other neuropsychiatric manifestations & No $=0 ;$ Yes $=1$ \\
\hline Active nephritis & $\mathrm{No}=0 ; \mathrm{Yes}=1$ \\
\hline Active nephrotic syndrome & No $=0 ;$ Yes $=1$ \\
\hline Active serositis & No $=0 ;$ Yes $=1$ \\
\hline Active inflammatory arthritis & No $=0 ;$ Yes $=1$ \\
\hline Active inflammatory rash & No $=0 ;$ Yes $=1$ \\
\hline Active mucosal ulcers & $\mathrm{No}=0 ;$ Yes $=1$ \\
\hline Alopecia & No $=0 ;$ Yes $($ acute $)=0.5 ;$ Yes $($ chronic $)=1$ \\
\hline Active vasculitis & No $=0 ;$ Yes $=1$ \\
\hline Hematologic disorder & $\mathrm{No}=0 ; \mathrm{Yes}=1$ \\
\hline Immunologic disorder & No $=0 ;$ Yes $=1$ \\
\hline Complement levels & $\begin{array}{l}\text { Normal } / \text { high }=0 ; \text { Low and negative dsDNA }=0.5 ; \text { Low and positive } \\
\text { dsDNA }=1\end{array}$ \\
\hline Sjögren syndrome & No $=0 ;$ Yes $=1$ \\
\hline Hypothyroidism & $\mathrm{No}=0 ; \mathrm{Yes}=1$ \\
\hline Hypertension & $\mathrm{No}=0 ; \mathrm{Yes}=1$ \\
\hline BMI & $\begin{array}{l}\text { BMI } 18.5-24.9 \mathrm{~kg} / \mathrm{m}^{2}=0 \text {; BMI } 25-29.9 \mathrm{~kg} / \mathrm{m}^{2}=0.5 \\
\text { BMI }<18.5 \text { or } \geq 30 \mathrm{~kg} / \mathrm{m}^{2}=1\end{array}$ \\
\hline Mood disorder & No $=0 ;$ Yes $=1$ \\
\hline Anxiety disorder & $\mathrm{No}=0 ; \mathrm{Yes}=1$ \\
\hline Headache disorder & No $=0 ;$ Yes $=1$ \\
\hline Self-rated health & Excellent $=0 ;$ Very good $=0.25 ;$ Good $=0.5 ;$ Fair $=0.75 ;$ Poor $=1$ \\
\hline Self-reported deterioration in health & Better or same $=0 ;$ Somewhat worse $=0.5 ;$ Much worse $=1$ \\
\hline Vigorous activities & Not limited at all $=0$; Somewhat limited $=0.5 ;$ Limited a lot $=1$ \\
\hline Moderate activities & Not limited at all $=0$; Somewhat limited $=0.5 ;$ Limited a lot $=1$ \\
\hline Lifting/carrying groceries & Not limited at all $=0$; Somewhat limited $=0.5 ;$ Limited a lot $=1$ \\
\hline Climbing stairs & Not limited at all $=0$; Somewhat limited $=0.5 ;$ Limited a lot $=1$ \\
\hline Bending, kneeling, or stooping & Not limited at all $=0$; Somewhat limited $=0.5$; Limited a lot $=1$ \\
\hline Walking $100 \mathrm{~m}$ & Not limited at all $=0$; Somewhat limited $=0.5$; Limited a lot $=1$ \\
\hline Bathing or dressing & Not limited at all $=0 ;$ Somewhat limited $=0.5 ;$ Limited a lot $=1$ \\
\hline Self-rated fatigue & $\begin{array}{l}\text { None }=0 ; \text { A little }=0.2 ; \text { Some }=0.4 ; \text { Moderate }=0.6 ; \text { Most }=0.8 \\
\text { Always }=1\end{array}$ \\
\hline Self-rated pain & $\begin{array}{l}\text { None }=0 ; \text { Very mild }=0.2 ; \text { Mild }=0.4 ; \text { Moderate }=0.6 ; \text { Severe }=0.8 ; \\
\text { Very severe }=1\end{array}$ \\
\hline
\end{tabular}

SLE: systemic lupus erythematosus; BMI: body mass index.

increasing age, from $19.3 \%$ (95\% CI 16.4-22.6) among patients < 30 years of age, to $28.1 \%$ (95\% CI 24.6-31.8) for patients aged $30-45$ years, and $38.5 \%$ (95\% CI $33.7-43.5 \%$ ) among patients aged 45 years or older. Very few patients $(\mathrm{n}=28 ; 1.7 \%)$ were classified as robust (SLICC-FI $\leq 0.03)$. These individuals were combined with the relatively less fit patients $(0.03<$ SLICC-FI $\leq 0.10)$ into a single category ("relatively fit"). 


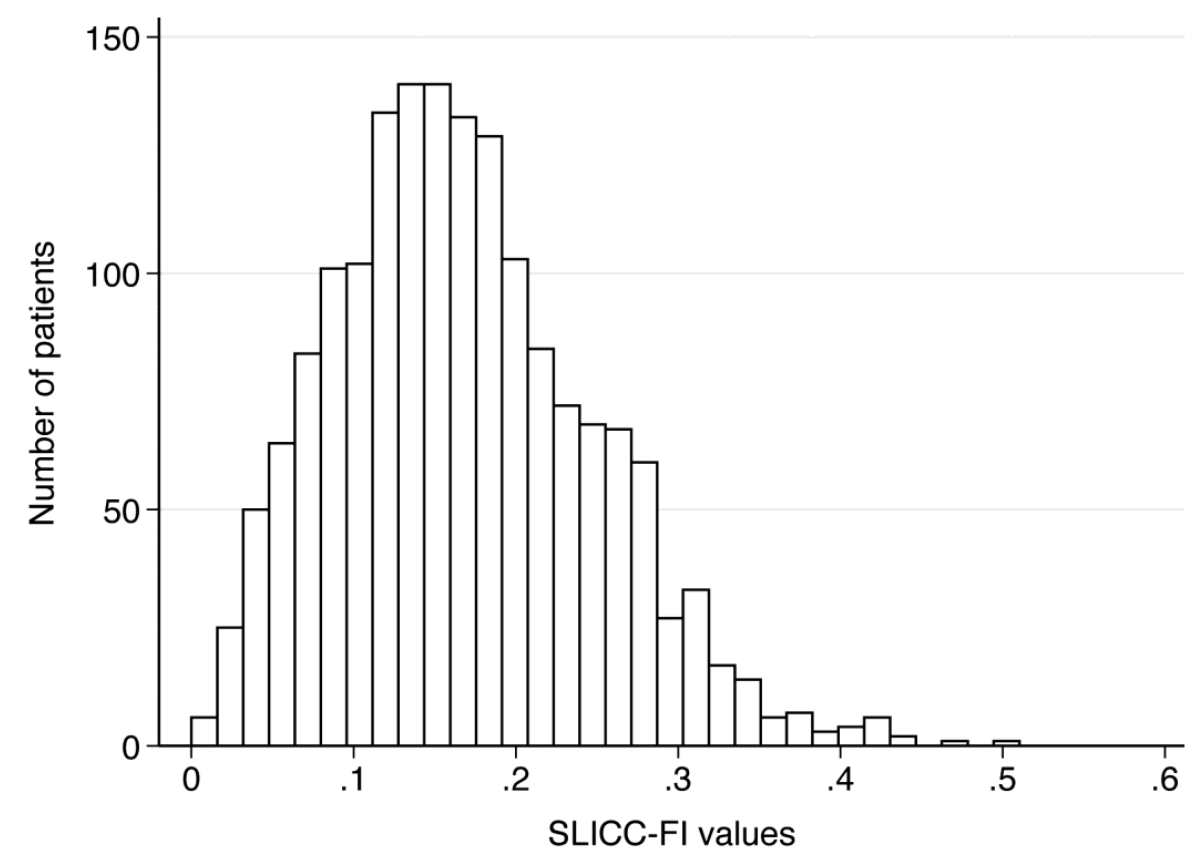

Figure 2. Distribution of SLICC-FI values among 1682 patients with SLE in the baseline dataset. SLICC: Systemic Lupus International Collaborating Clinics; FI: Frailty Index; SLE: systemic lupus erythematosus.

Table 4. Demographic characteristics of patients with SLE, stratified by baseline health status ${ }^{\mathrm{a}}(\mathrm{n}=1682)$.

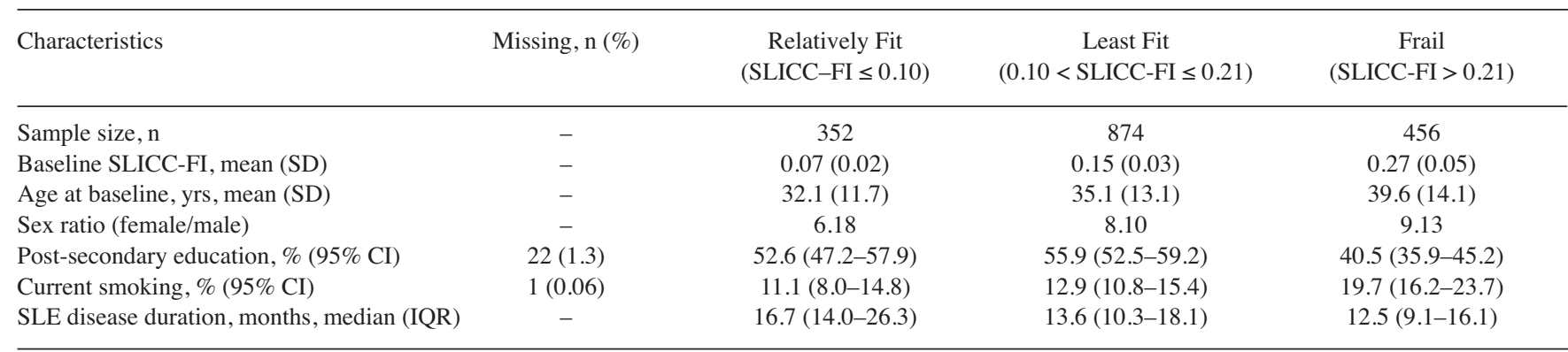

${ }^{a}$ Health status categories based on established FI cutpoints for the general population. IQR: interquartile range; SLICC: Systemic Lupus International Collaborating Clinics; FI: frailty index; SLE: systemic lupus erythematosus.

Compared to the relatively fittest patients, those who were classified as frail were older, less well educated, and more likely to be current smokers (Table 4). There was a trend toward a higher prevalence of frailty among women $(27.5 \%$; 95\% CI $25.3-29.9 \%)$ compared to men $(23.7 \%$; $95 \%$ CI $17.8-30.4 \%)$. There was also a trend toward shorter SLE disease duration among frail patients when compared to relatively fit patients.

Sensitivity analysis. Our results were similar when only patients with baseline assessments within 2 years of SLE diagnosis $(\mathrm{n}=1390)$ were considered (data not shown). Finally, SLICC-FI scores showed little sensitivity to which health deficits were included. In 100 iterations in which the SLICC-FI was recalculated using $80 \%$ of the 48 total deficits selected at random, the descriptive statistics and distribution of SLICC-FI scores were largely unchanged.

\section{DISCUSSION}

In this secondary analysis of data from the SLICC inception cohort, we have demonstrated the feasibility of constructing the first FI for patients with SLE. We have described the process for constructing the SLICC-FI in detail, including the selection of health deficits, and how these deficits were operationalized to calculate SLICC-FI values. We found a high prevalence of frailty among patients with SLE, the majority of whom were early in their disease course. A similar approach can be applied to investigate frailty in other SLE cohorts. However, additional studies are needed to demonstrate the validity of the SLICC-FI, including its association with the risk of future adverse health outcomes.

The process for constructing the SLICC-FI has many strengths. First, we followed a standard protocol ${ }^{20}$ to derive health deficits and their cutpoints from existing instruments 
that are well validated in SLE. With 48 items, the number of health deficits in the SLICC-FI is sufficient to provide stable and reliable estimates of frailty ${ }^{22,33,35,36}$. Last, the deficits in the SLICC-FI cover multiple organ systems and embrace both fixed and reversible health domains ${ }^{20}$.

That many small effects can aggregate to produce larger ones is well recognized in other disciplines. Applying this principle in medicine allows for the cumulative effect of multiple small deficits, which individually might not be statistically or clinically significant ${ }^{41}$. Some may be concerned about redundancy within the SLICC-FI and desire a more parsimonious list of items. However, each item contributes additional information, regardless of the correlation between them. One strength of the deficit accumulation approach to quantifying vulnerability is its ability to embrace the complexity of human systems, by placing less emphasis on specific items, and instead focusing on the overall effect of multiple health problems ${ }^{18}$. Indeed, similar to the results of prior work in other populations ${ }^{20,37}$, our sensitivity analysis demonstrated that SLICC-FI scores were not driven by a small number of specific variables but reflected the global effect of deficit accumulation.

The relationships that exist between deficits within the SLICC-FI are critical to its performance ${ }^{20}$. For example, the equal weighting of transient ischemic attacks and debilitating strokes in the "cerebrovascular disease" health deficit may appear to lack face validity, because these events clearly differ in their effect on overall health. However, an individual with a disabling stroke is more likely to have additional deficits related to their functional performance that will be reflected in their SLICC-FI score. Thus, including deficits related to functional status ensures that the health effect of different medical problems is accurately represented. Further, the potential reversibility of such deficits means that individuals may transition in and out of a frail state during followup, enabling the SLICC-FI to record improvements in a patient's status over time and distinguishing this instrument from the $\mathrm{SDI}^{30}$. Future work will examine the trajectories of SLICC-FI values during followup. Given that frailty is potentially treatable ${ }^{7}$, the SLICC-FI may be useful as an outcome measure for future intervention studies.

An alternative conceptual approach to the measurement of frailty is the Fried frailty phenotype ${ }^{19}$, which was recently evaluated in a prevalent cohort of 152 women with SLE ${ }^{42}$. In this study, $20 \%$ of the sample was classified as frail ${ }^{42}$. The presence of frailty was associated with increased risk of functional decline and mortality ${ }^{42}$, emphasizing its relevance in SLE. However, the authors also found that 2 of the 5 components of the frailty phenotype, as defined in geriatric medicine, had limited utility in $\mathrm{SLE}^{42}$, suggesting that measures with more relevance in SLE may be needed to better quantify frailty in this population.

There are several other challenges associated with applying the frailty phenotype in SLE that are overcome using the deficit accumulation approach. First, the frailty phenotype requires physical performance data ${ }^{18,19,42}$ that is not routinely collected in SLE and is unavailable in the SLICC inception cohort. In contrast, the variables in the SLICC-FI are derived from existing validated instruments that are commonly used in SLE cohorts and rheumatology clinics, allowing the SLICC-FI to be easily implemented in other clinical and research settings. Another limitation of the frailty phenotype is its lack of granularity, because individuals are assigned to 1 of 3 risk categories ${ }^{18,19}$. Meanwhile, the SLICC-FI identifies a full spectrum of vulnerability, and studies using this approach in other populations have demonstrated a dose-response relationship between FI values and risk of adverse outcomes $20,22,23,33$. Finally, with only 5 variables included in the frailty phenotype, modifying how the phenotypic criteria are defined can alter the prevalence estimates for frailty considerably 43 . In contrast, the properties of the FI remain remarkably consistent regardless of the number or type of variables included $20,22,23,24,33$. While the FI and the frailty phenotype have shown reasonable agreement in geriatric populations ${ }^{34,37}$, it is unclear whether this correlation exists in SLE. Future work should investigate agreement between the SLICC-FI and the Fried phenotype for the identification of frailty in SLE.

In our study, $27.1 \%$ of patients were classified as frail. This is higher than expected for similarly aged individuals in the general population ${ }^{15,32,44}$. For example, among patients with SLE who are $<30$ years of age, $19.3 \%$ were classified as frail, compared with an estimated frailty prevalence of $2.0 \%$ among Canadian adults in the same age group $^{15}$. SLICC-FI values (mean FI 0.17 ) were substantially lower than FI scores reported in other clinical cohorts, including patients with human immunodeficiency virus (mean FI 0.31$)^{22}$ and systemic sclerosis (mean FI 0.33$)^{23}$. This could be partially explained by the higher mean age in these other cohorts, as deficits accumulate with increasing age $^{35}$. Overall, our findings support those of prior studies in non-SLE populations that have demonstrated older age, female sex, lower educational attainment, and cigarette smoking to be associated with higher prevalence of frailty $15,20,33$

There is biological plausibility to our findings. The link between chronic inflammation and frailty is well established, with elevated markers of systemic inflammation observed among frail older adults compared with those who are not frail $^{45}$. Further, certain inflammatory cytokines, such as interleukin 6 , have been implicated in the pathogenesis of both frailty and SLE ${ }^{45,46}$. While more work is required to fully elucidate the role of immune dysregulation in the development of frailty, this could represent a potential mechanism for accelerated aging in SLE.

Our study has important limitations. Because of missing data, we were unable to calculate SLICC-FI scores at

Personal non-commercial use only. The Journal of Rheumatology Copyright @ 2019 . All rights reserved. 
enrollment for some patients. Despite this, $82.6 \%$ of eligible patients had their baseline assessment for SLICC-FI construction within 2 years of SLE diagnosis. Second, our sample size is small compared with some other FI studies $15,20,33$, but is still sufficient for FI construction ${ }^{23}$. Third, we used FI cutpoints derived from general population samples to estimate the prevalence of frailty in our dataset $15,37,38$. It is possible that a different cutoff for SLICC-FI scores should be used to define phenotypic frailty in SLE. This is an area for future research. Last, we have constructed the SLICC-FI in a cohort of relatively young, recently diagnosed patients with SLE. It remains unclear whether these findings can be generalized to older patients with longstanding SLE. Prior to use, validation of the SLICC-FI is required, including external validation in prevalent SLE cohorts and confirmation of its association with the risk of future adverse health outcomes.

Evaluating frailty through deficit accumulation provides a novel approach to the quantification of vulnerability among patients with SLE. We identified a high prevalence of frailty among patients with SLE, which warrants additional investigation. The SLICC-FI requires validation prior to its use as a tool to identify patients with SLE who are at increased risk for adverse outcomes.

\section{ONLINE SUPPLEMENT}

Supplementary material accompanies the online version of this article.

\section{REFERENCES}

1. Tsokos GC. Systemic lupus erythematosus. N Engl J Med 2011;365:2110-21

2. Urowitz MB, Gladman DD, Tom B, Ibanez D, Farewell VT. Changing patterns in mortality and disease outcomes for patients with systemic lupus erythematosus. J Rheumatol 2008;35:2152-8.

3. Sutton EJ, Davidson JE, Bruce IN. The systemic lupus international collaborating clinics (SLICC) damage index: a systematic literature review. Semin Arthritis Rheum 2013;43:352-61.

4. Yurkovich M, Vostretsova K, Chen W, Aviña-Zubieta JA. Overall and cause-specific mortality in patients with systemic lupus erythematosus: a meta-analysis of observational studies. Arthritis Care Res 2014;66:608-16.

5. Fors Nieves CE, Izmirly PM. Mortality in systemic lupus erythematosus: an updated review. Curr Rheumatol Rep 2016;18:21.

6. Rahman P, Gladman D, Urowitz MB, Hallett D, Tam LS. Early damage as measured by the SLICC/ACR damage index is a predictor of mortality in systemic lupus erythematosus. Lupus 2001;10:93-6.

7. Clegg A, Young J, Iliffe S, Rikkert MO, Rockwood K. Frailty in elderly people. Lancet 2013;381:752-62.

8. Partridge JS, Harari D, Dhesi JK. Frailty in the older surgical patient: a review. Age Ageing 2012;41:142-7.

9. Bao Y, Dalrymple L, Chertow GM, Kaysen GA, Johansen KL. Frailty, dialysis initiation, and mortality in end-stage renal disease. Arch Intern Med 2012;172:1071-7.

10. Lai JC, Feng S, Terrault NA, Lizaola B, Hayssen H, Covinsky K. Frailty predicts waitlist mortality in liver transplant candidates. Am J Transplant 2014;14:1870-9.

11. Abel GA, Klepin HD. Frailty and the management of hematologic malignancies. Blood 2018;131:515-24.
12. Denfeld QE, Winters-Stone K, Mudd JO, Gelow JM, Kurdi S, Lee CS. The prevalence of frailty in heart failure: a systematic review and meta-analysis. Int J Cardiol 2017;236:283-9.

13. Muscedere J, Waters B, Varambally A, Bagshaw SM, Boyd JG, Maslove D, et al. The impact of frailty on intensive care unit outcomes: a systematic review and meta-analysis. Intensive Care Med 2017;43:1105-22.

14. Fulop T, Larbi A, Witkowski JM, McElhaney J, Loeb M, Mitnitski A, et al. Aging, frailty and age-related diseases. Biogerontology 2010;11:547-63.

15. Rockwood K, Song X, Mitnitski A. Changes in relative fitness and frailty across the adult lifespan: evidence from the Canadian National Population Health Survey. CMAJ 2011;183:E487-94.

16. Brothers TD, Kirkland S, Guaraldi G, Falutz J, Theou O, Johnston BL, et al. Frailty in people aging with human immunodeficiency virus (HIV) infection. J Infect Dis 2014;210:1170-9.

17. Ness KK, Armstrong GT, Kundu M, Wilson CL, Tchkonia T, Kirkland JL. Frailty in childhood cancer survivors. Cancer 2015;121:1540-7.

18. Theou O, Walston J, Rockwood K. Operationalizing frailty using the frailty phenotype and deficit accumulation approaches. Interdiscipl Top Gerontol 2015;41:66-73.

19. Fried LP, Tangen CM, Walston J, Newman AB, Hirsch C, Gottdiener J, et al; Cardiovascular Health Study Collaborative Research Group. Frailty in older adults: evidence for a phenotype. J Gerontol A Biol Sci Med Sci 2001;56A:M146-56.

20. Searle SD, Mitnitski A, Gahbauer EA, Gill TM, Rockwood K. A standard procedure for creating a frailty index. BMC Geriatr 2008;8:24.

21. Mitnitski A, Rockwood K. Aging as a process of deficit accumulation: its utility and origin. Interdiscipl Top Geronto 2015;40:85-98.

22. Guaraldi G, Brothers TD, Zona S, Stentarelli C, Carli F, Malagoli A, et al. A frailty index predicts survival and incident multimorbidity independent of markers of HIV disease severity. AIDS 2015;29:1633-41.

23. Rockwood MR, MacDonald E, Sutton E, Rockwood K, Scleroderma Research Group, Baron M; Canadian Scleroderma Research Group. Frailty index to measure health status in people with systemic sclerosis. J Rheumatol 2014;41:698-705.

24. Kojima G, Iliffe S, Walters K. Frailty index as a predictor of mortality: a systematic review and meta-analysis. Age Ageing 2018;47:193-200.

25. Hubbard RE, Peel NM, Smith M, Dawson B, Lambat Z, Bak M, et al. Feasibility and construct validity of a Frailty index for patients with chronic kidney disease. Australas J Ageing 2015;34:E9-12.

26. Hochberg MC. Updating the American College of Rheumatology revised criteria for the classification of systemic lupus erythematosus. Arthritis Rheum 1997;40:1725.

27. The American College of Rheumatology nomenclature and case definitions for neuropsychiatric lupus syndromes. Arthritis Rheum 1999;42:599-608.

28. Hanly JG, Urowitz MB, Sanchez-Guerrero J, Bae SC, Gordon C, Wallace DJ, et al; Systemic Lupus International Collaborating Clinics. Neuropsychiatric events at the time of diagnosis of systemic lupus erythematosus: an international inception cohort study. Arthritis Rheum 2007;56:265-73.

29. Gladman DD, Ibañez D, Urowitz MB. Systemic lupus erythematosus disease activity index 2000. J Rheumatol 2002;29:288-91.

30. Gladman D, Ginzler E, Goldsmith C, Fortin P, Liang M, Urowitz M, et al. The development and initial validation of the Systemic Lupus International Collaborating Clinics/American College of Rheumatology damage index for systemic lupus erythematosus. Arthritis Rheum 1996;39:363-9. 
31. Ware JE, Sherbourne CD. The MOS 36-item short-form health survey (SF-36). I. Conceptual framework and item selection. Med Care 1992;30:473-83.

32. Rockwood K, Blodgett JM, Theou O, Sun MH, Feridooni HA, Mitnitski A, et al. A frailty index based on deficit accumulation quantifies mortality risk in humans and in mice. Sci Rep 2017;7:43068

33. Mitnitski A, Song X, Skoog I, Broe GA, Cox JL, Grunfeld E, et al. Relative fitness and frailty of elderly men and women in developed countries and their relationship with mortality. J Am Geriatr Soc 2005;53:2184-9.

34. Kulminski AM, Ukraintseva SV, Kulminskaya IV, Arbeev KG, Land $\mathrm{K}$, Yashin AI. Cumulative deficits better characterize susceptibility to death in elderly people than phenotypic frailty: lessons from the Cardiovascular Health Study. J Am Geriatr Soc 2008;56:898-903.

35. Rockwood K, Mitnitski A. Frailty defined by deficit accumulation and geriatric medicine defined by frailty. Clin Geriatr Med 2011;27:17-26.

36. Theou O, Brothers TD, Mitnitski A, Rockwood K. Operationalization of frailty using eight commonly used scales and comparison of their ability to predict all-cause mortality. J Am Geriatr Soc 2013;61:1537-51.

37. Rockwood K, Andrew M, Mitnitski A. A comparison of two approaches to measuring frailty in elderly people. J Gerontol A Biol Sci Med Sci 2007;62A:738-43.

38. Rockwood K, Song X, MacKnight C, Bergman H, Hogan DB, McDowell I, et al. A global clinical measure of fitness and frailty in elderly people. CMAJ 2005;173:489-95.
39. Rockwood K, Mitnitski A, Song X, Steen B, Skoog I. Long-term risks of death and institutionalization of elderly people in relation to deficit accumulation at age 70. J Am Geriatr Soc 2006;54:975-9.

40. Flegal KM, Graubard BI, Williamson DF, Gail MH. Cause-specific excess deaths associated with underweight, overweight, and obesity. JAMA 2007;298:2028-37.

41. Rutenberg AD, Mitnitski AB, Farrell SG, Rockwood K. Unifying aging and frailty through complex dynamical networks. Exp Gerontol 2018;107:126-9.

42. Katz PP, Andrews J, Yazdany J, Schmajuk G, Trupin L, Yelin E. Is frailty a relevant concept in SLE? Lupus Sci Med 2017;4:e000186.

43. Theou O, Cann L, Blodgett J, Wallace LM, Brothers TD, Rockwood K. Modifications to the frailty phenotype criteria: Systematic review of the current literature and investigation of 262 frailty phenotypes in the Survey of Health, Ageing, and Retirement in Europe. Ageing Res Rev 2015;21:78-94.

44. Mitnitski A, Rockwood K. The rate of aging: the rate of deficit accumulation does not change over the adult life span. Biogerontology 2016;17:199-204.

45. Soysal P, Stubbs B, Lucato P, Luchini C, Solmi M, Peluso R, et al. Inflammation and frailty in the elderly: a systematic review and meta-analysis. Ageing Res Rev 2016;31:1-8.

46. Jacob N, Stohl W. Cytokine disturbances in systemic lupus erythematosus. Arthritis Res Ther 2011;13:228. 